\title{
Dynamic human resource selection for business process exceptions
}

\section{Jooseok Lee (1) | Seunghoon Lee (D) | Jinwoo Kim I Injun Choi}

Department of Industrial and Management Engineering, Pohang University of Science and Technology (POSTECH), Pohang, South Korea

\section{Correspondence}

Injun Choi, Department of Industrial and Management Engineering, Pohang University of Science and Technology (POSTECH), Namgu, Pohang 37673, South Korea.

Email: injun@postech.ac.kr

Funding information

Korea Small and Medium Business Administration, Grant/Award Number: 10031496; National Research Foundation of Korea(NRF), Grant/Award Number:

NRF-2017R1A2B4008029

\begin{abstract}
A key capability of today's organizations is to flexibly and effectively react to unexpected events. A critical case of an unexpected event is sudden unavailability of human resources, which was not properly addressed by existing resource allocation approaches. This paper proposes a systematic approach that analyzes event logs to select suitable substitutes if the initial human resources become unavailable. The approach uses process mining and social network analysis to derive a metric called degree of substitution, which measures how much the work experiences of the human resources overlap, from the two perspectives: task execution and transfer of work. Along with the metric, suitable substitutes are also identified. A simulation demonstrates that the approach identifies suitable substitutes more effectively and accurately than existing allocation methods such as role-based allocation or random allocation. The proposed approach will increase the effectiveness of dynamic allocation of human resources, especially in an exceptional situation.
\end{abstract}

\section{1 | INTRODUCTION}

During execution of business processes, numerous exceptions can occur. For example, core human resources can become unavailable. Today's organizations must react appropriately to these situations to ensure that their processes are executed successfully (Dumas, La Rosa, Mendling, \& Reijers, 2013; Kim, Choi, \& Park, 2011; Kim, Lee, Lee, \& Choi, 2017; Reichert \& Weber, 2012). Responses to such exceptions include trying alternative paths, adding supplementary behaviors, and changing the resource allocation (Reichert \& Weber, 2012). The last type of response is especially important when exceptions occur in business processes in which human performers have important roles. It includes delegation, escalation, deallocation, and reallocation of work items (Russell, van der Aalst, Hofstede, \& Edmond, 2005).

Many business process management systems, however, use primitive approaches such as the "push or pull" mechanism to distribute work items to performers (Huang, van der Aalst, Lu, \& Duan, 2010; Liu, Cheng, \& Ni, 2012; Liu, Wang, Yang, \& Sun, 2008). Thus, work items are often assigned to "too few, too many, or even the wrong set of performers" (Kumar, van der Aalst, \& Verbeek, 2002). A critical case is that a work item is assigned to a performer who is absent or overloaded. Some research has proposed advanced human-resource allocation mechanisms to avoid these errors. However, the mechanisms do not consider exceptional situations such as sudden unavailability of the performers. Such situations cannot all be anticipated and incorporated in the allocation mechanism in advance, so appropriate substitutes must be identified quickly. That is, alternative performers who can minimize the impact of the exceptions must be identified; this task has not been properly addressed by previous studies.

Furthermore, existing studies do not consider the perspective of transfer of work, which can significantly affect the efficiency of business processes (Kumar, Dijkman, \& Song, 2013). Transfer of work, refers to passing work items from one task to the next, often contributes a significant portion of delays in execution of business processes (Dustdar \& Hoffmann, 2007; Hearn \& Choi, 2013; Lee, Sung, Song, \& Choi, 2015). Accordingly, both task execution and transfer of work must be considered.

This paper proposes a systematic approach to rapidly identify suitable substitutes by considering task execution and transfer of work. Specifically, the approach uses a metric called degree of substitution (DoS) to quantify the overlap in the work experiences of human resources. DoS is derived using process mining techniques and social network. The approach also constructs a substitution network to visualize the DoS. A simulation demonstrates that the approach identifies 
suitable substitutes more effectively and accurately than existing allocation mechanisms such as role-based allocation or random allocation.

The remainder of this paper is organized as follows. Section 2 discusses existing works related to human resource allocation in the context of business process management. Section 3 introduces the approach to identifying suitable substitutes and describes the details of applied techniques. Section 4 presents a case study along with a simulation result to demonstrate the applicability and feasibility of the presented approach. Section 5 discusses practical implications. Section 6 concludes the paper.

\section{I RELATED WORK}

Effective allocation of human resources can significantly affect the performance of business processes (Cabanillas et al., 2013; Huang, Lu, \& Duan, 2011; Ly, Rinderle, Dadam, \& Reichert, 2006; Wibisono, Nisafani, Bae, \& Park, 2016), so it is becoming increasingly important in business process management. However, most existing studies have focused on the control-flow perspective and have ignored the humanresource perspective (Russell et al., 2005; Song \& van der Aalst, 2008). Further, to allocate human resources, current business process management systems apply rather primitive mechanisms, such as the "pull or push" mechanism. As a result, human resources are often allocated to inappropriate tasks, especially when unexpected exceptions occur. Advanced behavioral requirements, such as escalation, delegation, reallocation, and deallocation, are identified to respond appropriately to those exceptional situations (Russell et al., 2005), but few approaches support these advanced behavioral requirements (Reichert \& Weber, 2012; Russell et al., 2005).

Several studies proposed advanced mechanisms to increase the effectiveness and flexibility of resource allocation. One dynamic work-distribution method uses four parameters-availability, conformance, urgency, and suitability (Kumar et al., 2002)-to direct work items to less-qualified human resources if qualified resources are not available. Supervised machine learning can be used for semiautomatic human-resource allocation (Liu et al., 2008); this method recommends suitable human resources by examining the patterns of previous activities that are recorded in event logs. A decision-tree learning algorithm can be used to extract meaningful resource allocation rules from event logs and organizational information (e.g., an organizational model; Ly et al., 2006). Association rule mining has been used to learn meaningful resource allocation rules from event logs (Huang et al., 2011; Liu et al., 2012). An adaptive resource allocation mechanism that uses reinforcement learning can derive the most suitable allocation rule in a given process context (Huang et al., 2010). The efficiency of human resources varies over time; Bayesian-based allocation mechanism can be used to represent the variance (Wibisono et al., 2016).

Most existing studies focused on incorporating all possible dynamics into the proposed allocation mechanisms, that is, on finding the most suitable human resources in a given context. These studies did not consider exceptional situations. The full set of all exceptional situations cannot be anticipated, so a systematic method to find alternative performers must be developed.
Further, most previous studies have not considered the perspective of transfer of work, although it constitutes a significant proportion of delays in executing business processes. Incorporating the effect of transfer of work into allocation mechanisms can maximize cooperation between human resources (Kumar et al., 2013), but this approach also does not consider exceptional situations. The first proposed approach to address this research gap used event logs to derive suitable substitutes (Lee, Lee, Kim, \& Choi, 2016). The present paper extends the previous work with a case study and simulation to demonstrate the superiority of the approach over existing allocation mechanisms.

\section{3 | APPROACH TO SELECTING SUITABLE ALTERNATIVE PERFORMERS}

The approach considers two perspectives when seeking suitable alternative performers: task execution and transfer of work. Task execution indicates whether a certain substitute human resource for an initial performer can successfully execute a given task. Transfer of work indicates whether the substitute human resource can successfully pass a task to a successor who will perform a subsequent task or can receive a task from a predecessor who performed the preceding task (Hearn \& Choi, 2013).

The proposed approach assumes that a human resource can be most effectively replaced by a person who works on similar tasks or similar transfer of work, rather than by a person who does completely different tasks. Each human resource has a "profile" which will be detailed in a later section, based on how frequently he or she conducts a specific task or transfer of work. Other criteria, such as performance, quality, cost, expertise, or workload (Arias, Rojas, Munoz-Gama, \& Sepúlveda, 2015), may also be used to define the profile of human resources.

The proposed approach applies process mining and social network analysis (SNA) techniques to find the most suitable substitutes. Process mining extracts valuable process-related information such as process models (Van der Aalst, 2011) from event logs; this approach uses organizational mining, which specializes in extracting organizational information (Song \& van der Aalst, 2008). SNA primarily focuses on analyzing relationships between actors in a given social network (Wasserman \& Faust, 1994). In particular, a concept of structural equivalence, which quantifies the structural similarity of actors in a given social network, is used in the approach.

\subsection{Definition of event logs}

Current business process management systems log all kinds of process-related events, such as an initiation and completion of tasks. Event logs (Table 1; Song \& van der Aalst, 2008) may contain useful information such as instance ID, name of the executed task, designated performer, or time stamp. Each row of the table corresponds to an event and contains information of instance (or case) ID, task name, and performer. Event logs may need preprocessing due to incomplete information or noise (Van der Aalst, 2011). 
TABLE 1 Example of event logs; each row corresponds to an event and contains information of instance (or case) ID, task name, and performer

\begin{tabular}{lll} 
Case & Task & Performer \\
\hline 1 & Task A & P 1 \\
2 & Task A & P 2 \\
1 & Task B & P 2 \\
\hline 3 & Task B & P 3 \\
\hline 2 & Task C & P 4 \\
2 & Task D & P 4 \\
\hline 1 & Task C & P 3 \\
$\ldots$ & $\ldots$ & $\ldots$ \\
\hline
\end{tabular}

\section{2 | Deriving profiles of human resources}

Human resources' task-execution profiles can be derived from event logs by using a metric based on joint activities (Van der Aalst, Reijers, \& Song, 2005). This metric shows how frequently each human resource conducts a certain task. The formal definition of this metric is as follows. Let $\mathrm{R}=\left\{r_{1}, r_{2}, \ldots r_{n}\right\}$ be a set of human resources, $T=\left\{t_{1}, t_{2}, \ldots t_{m}\right\}$ be a set of tasks, and $c=\left\{c_{0}, c_{1}, \ldots\right\} \in L$ be a trace of an event log, or a case, in a business process. Function $\pi_{t}$ takes an event $c_{i}$ as an input and returns an associated task $t_{i}$. Function $\pi_{p}$ takes event $c_{i}$ as input and returns an associated performer $r_{j}$. Then the metric based on joint activities is defined as follows:

$$
r_{a} \Delta_{L} t_{b}=\sum_{c \in L} \sum_{0 \leq i<|c|}\left\{\begin{array}{cc}
1 & \text { if } \pi_{t}\left(c_{i}\right)=t_{a} \text { and } \pi_{p}\left(c_{i}\right)=r_{b} \\
0 & \text { otherwise. }
\end{array}\right.
$$

The metric is expressed as a human resource-by-task matrix (Table 2), which represents how frequently each human resource conducts a certain task. For example, human resource R1 conducted task T1 64 times and task T2 five times.

A profile of transfer-of-work can be derived in a similar way. In this case, a handover metric (Van der Aalst et al., 2005) is used. The metric shows how frequently two human resources send and receive work items to and from each other. The formal definition of the metric is as follows. $\pi_{t}$ and $\pi_{p}$, are also used in this definition. Additionally, a symbol $\rightarrow$ denotes a causality relationship between two successive tasks in a business process model. The handover metric between two human resources can be defined as follows:

$r_{\mathrm{a}} \triangleright_{L} r_{\mathrm{b}}=\sum_{c \in L} \sum_{0 \leq i<|c|-1} \begin{cases}1 & \pi_{p}\left(c_{i}\right)=r_{a} \text { and } \pi_{p}\left(c_{i+1}\right)=r_{b} \text { and } \pi_{t}\left(c_{i}\right) \rightarrow \pi_{t}\left(c_{i+1}\right) \\ 0 & \text { otherwise. }\end{cases}$

The handover metric can be expressed as a human resource-byhuman resource matrix (Table 3); each row indicates how frequently

TABLE 2 Example of a task execution matrix

\begin{tabular}{rrrrr} 
& Task A & Task B & Task C & Task D \\
R1 & 64 & 5 & 0 & 0 \\
R2 & 87 & 11 & 0 & 0 \\
R3 & 0 & 8 & 28 & 0 \\
R4 & 0 & 0 & 1 & 44 \\
\hline
\end{tabular}

TABLE 3 Example of a transfer-of-work matrix

\begin{tabular}{lllll|} 
& R1 & R2 & R3 & R4 \\
R1 & 5 & 0 & 22 & 2 \\
R2 & 2 & 3 & 18 & 1 \\
\hline R3 & 10 & 0 & 0 & 27 \\
R4 & 3 & 0 & 20 & 8 \\
\hline
\end{tabular}

each human resource sends work items to others, and each column indicates how frequently each human resource received work items from others. For example, R1 sent work items to R2 zero times, to R3 22 times, and to R4 twice. R1 sent work items to her/himself five times. Likewise, R1 received work items from her/himself five times, from R2 twice, from R3 10 times, and from R4 three times.

\section{3 | Assessing the substitutability with degree of substitution}

The approach interprets the profiles of human resources as social networks and applies SNA, with particular attention to structural equivalence (Song \& van der Aalst, 2008). Structural equivalence measures a structural similarity of nodes in a network. For example, nodes $i$ and $j$ are structurally equivalent if $i$ has relations with node $k$, if and only if $j$ also has relations with node $k$ for all node $k$ and relations (Wasserman \& Faust, 1994); that is, the concept of structural similarity corresponds to the similarity of profiles.

Various measures such as Pearson's correlation, Euclidean distance, or Jaccard coefficient (Hanneman \& Riddle, 2005; Wasserman $\&$ Faust, 1994) have been proposed to quantify structural similarity. The Jaccard coefficient is defined as the ratio of the intersection of samples sets to their union. The approach uses a modified Jaccard coefficient to measure the similarity of profiles of human resources. The Jaccard coefficient can lead to biased results because it uses the maximum and minimum values of each profile. For example, if certain values in profiles are extremely larger than others, the value of Jaccard similarity will be biased. This section introduces a modified Jaccard coefficient to avoid the problem.

The formal definition, also referred to as a DoS, is as follows. Let $P=\left\{p_{1}, p_{2}, \ldots p_{n}\right\}$ and $Q=\left\{q_{1}, q_{2}, \ldots q_{n}\right\}$ be profiles of two human resources, where $\mathrm{P}$ is a performer to be replaced. Let $x_{i}$ be a ratio of the $i^{\text {th }}$ values of $P$ and $Q$ 's profiles where $y_{i}$ be an indicator that represents whether the $i^{\text {th }}$ value of P's profile is $>0$. Then Q's DoS relative to $P$ is defined as follows:

$$
\begin{aligned}
\operatorname{DoS}(\mathrm{P}, \mathrm{Q}) & =\frac{\sum_{i} x_{i}}{\sum_{i} y_{i}}, \\
\text { where } x_{i} & =\left\{\begin{array}{cc}
1 & \text { if } q_{i}>p_{i} \\
q_{i} / p_{i} & \text { otherwise }
\end{array} \text { and } y_{i}=\left\{\begin{array}{cc}
1 & \text { if } p_{i}>0 \\
0 & \text { otherwise. }
\end{array}\right.\right.
\end{aligned}
$$

DoS can be represented in two types of human resource-byhuman resource matrix; one (Table 4) is based on the task-execution perspective $\left(\operatorname{DoS}_{\mathrm{TE}}\right)$, and one (Table 5 ) is based on the transfer-ofwork perspective $\left(\mathrm{DoS}_{\mathrm{TW}}\right)$. A high DoS indicates a high overlap in work experiences. For example, R2 is the most suitable substitute for R1 for task execution, because R2 has the highest value among 
TABLE 4 Degree of substitution matrix (task execution)

\begin{tabular}{lcc} 
& In-degree & Out-degree \\
R1 & 0.305 & 0.440 \\
R2 & 0.296 & 0.369 \\
R3 & 0.540 & 0.147 \\
R4 & 0.011 & 0.196 \\
\hline
\end{tabular}

TABLE 5 Degree of substitution matrix (transfer of work)

\begin{tabular}{lllll} 
& R1 & R2 & R3 & R4 \\
R1 & 1 & 0.579 & 0.714 & 0.773 \\
R2 & 0.746 & 1 & 0.571 & 0.762 \\
R3 & 0.212 & 0.091 & 1 & 0.229 \\
R4 & 0.714 & 0.498 & 0.714 & 1 \\
\hline
\end{tabular}

possible substitutes (Table 4). Likewise, R4 is the most suitable substitute for R1 in terms of transfer of work according to Table 5. The matrices are not symmetric; this trait confirms the intuition that a highly-experienced human resource can successfully substitute for one with little experience but not vice versa.

Finally, various averaging techniques can be applied to aggregate the two perspectives.

$$
\operatorname{DoS}_{\mathrm{Both}}=\frac{2 \mathrm{DoS}_{\mathrm{TE} *} \operatorname{DoS}_{\mathrm{TW}}}{\operatorname{DoS}_{\mathrm{TE}}+\operatorname{DoS}_{\mathrm{TW}}}
$$

The harmonic mean of the degree of substitution is used to find substitutes who are superior in both perspectives. The balance between the two perspectives can be adjusted according to the business process.

\section{4 | Constructing substitution networks}

Finally, the DoS between human resources is visualized in a network form called substitution network, which shows a holistic view of the substitutability between human resources (Figure 1). In the graph, each node indicates a human resource, and each arc indicates the DoS between two nodes. The thickness of an arc increases as the

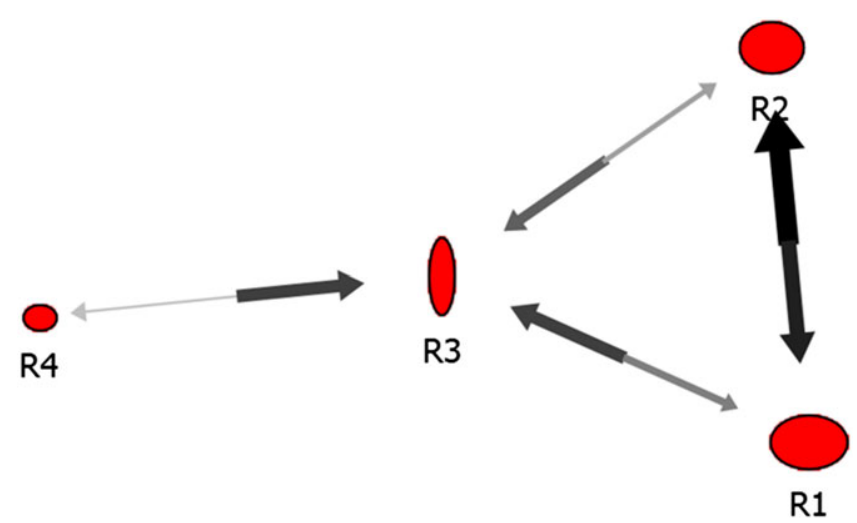

FIGURE 1 An example of substitution network [Colour figure can be viewed at wileyonlinelibrary.com]
DoS increases. The direction of the arc implies the roles of each human resource connected (e.g., the node to which the arrow points is the potential substitute). For example, R2 and R3 are two potential substitutes for R1, and R2 is better than R3.

The proposed approach simplifies the substitution network by eliminating relationships that have DoS less than some threshold; this technique is also useful to facilitate visualization of complex networks. The substitution network can also be related to an organizational model to increase the value of the implications. For example, nodes in the network can be projected onto hierarchical levels or organizational departments, and a new substitution network can be constructed in which the nodes correspond to organizational entities (e.g., roles or departments).

To derive more insightful implications, out-degrees or in-degrees of human resources can be calculated and analyzed (Table 6). For example, an out-degree of a human resource, or a weighted sum of arcs directed from her/him, indicates the number of potential substitutes who can replace her/him: The human resource has many substitutes if she/he has a high out-degree. On the contrary, a human resource with a low out-degree can be considered as a critical resource in the organization because few substitutes exist (Cabanillas, Resinas, del-Río-Ortega, \& Ruiz-Cortés, 2015). An in-degree of a human resource indicates his/her capability to replace other performers. A human resource who has a high in-degree is a multi-player who is competent at many tasks.

\section{5 | Selecting suitable alternative performers}

The proposed DoS approach rapidly identifies suitable alternative performers in case of exceptions, such as sudden unavailability of process performers or increase in workload (Figure 2). For example, if a performer becomes unavailable due to illness, the approach uses event logs to calculate the DoS and identify the most suitable substitutes. Then a manager decides who will be the actual alternative performer. If a workload suddenly increases, the procedure of selecting substitutes is slightly different. First, a copy of a new instance of the overloaded task must be created. Then, based on DoS, the approach identifies a suitable substitute and allocates him/her to the newly created instance.

\section{4 | CASE STUDY AND SIMULATION}

\section{1 | Case study}

This section presents a case study to demonstrate the applicability of the proposed approach. Event logs are analyzed in the case study of a

TABLE 6 Example of in- and out-degree

$\begin{array}{lll} & \text { In-degree } & \text { Out-degree } \\ \text { R1 } & 1 & 0.579 \\ \text { R2 } & 0.746 & 1 \\ \text { R3 } & 0.212 & 0.091 \\ \text { R4 } & 0.714 & 0.498\end{array}$


Usage scenario 1 : Unavailability of the initial process performer

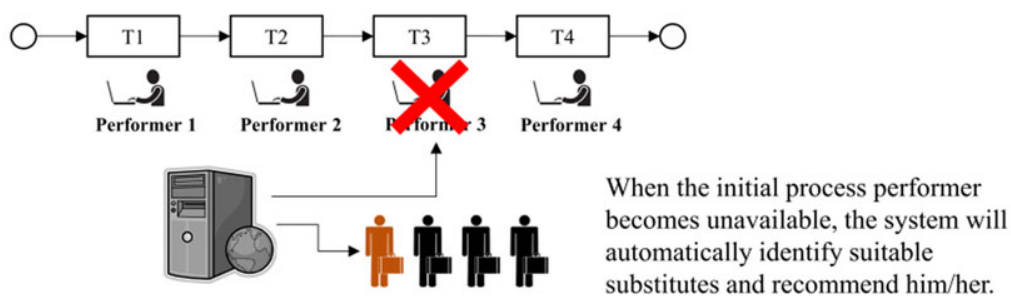

Usage scenario 2 : Sudden increase of workload
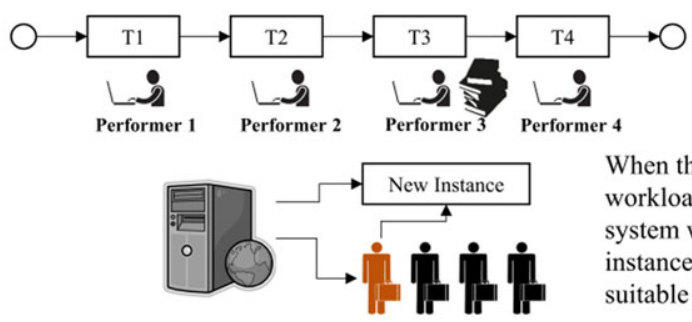

When there is a sudden increase of workload for particular performer, the system will automatically create new instance of his/her task and assign suitable substitute to it.

FIGURE 2 An illustration of the usage scenarios [Colour figure can be viewed at wileyonlinelibrary.com]

purchasing process, which consists of eight activities (Figure 3), using business process model and notation. The eight activities are "Create Purchase Requisition," "Analyze Purchase Requisition," "Create Request for Quotation Requester Manager," "Create Request for Quotation Requester," "Analyze Request for Quotation," "Send Request for Quotation to Supplier," "Create Quotation Comparison Map," and
"Analyze Quotation Comparison Map." Twenty human resources are involved in the process.

The approach derived two types of profiles from event logs that contain 3,880 events and 608 cases or instances. Then the profiles are used to calculate DoS in the perspectives of task execution and transfer of work. A substitution network (Figure 4)

FIGURE 3 A purchasing process.

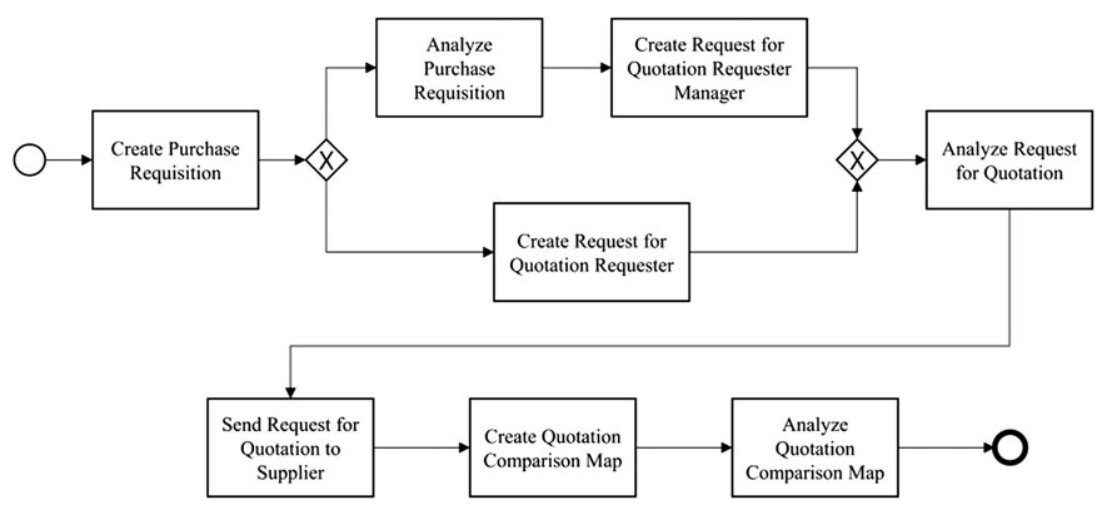

FIGURE 4 The substitution network [Colour figure can be viewed at wileyonlinelibrary.com]
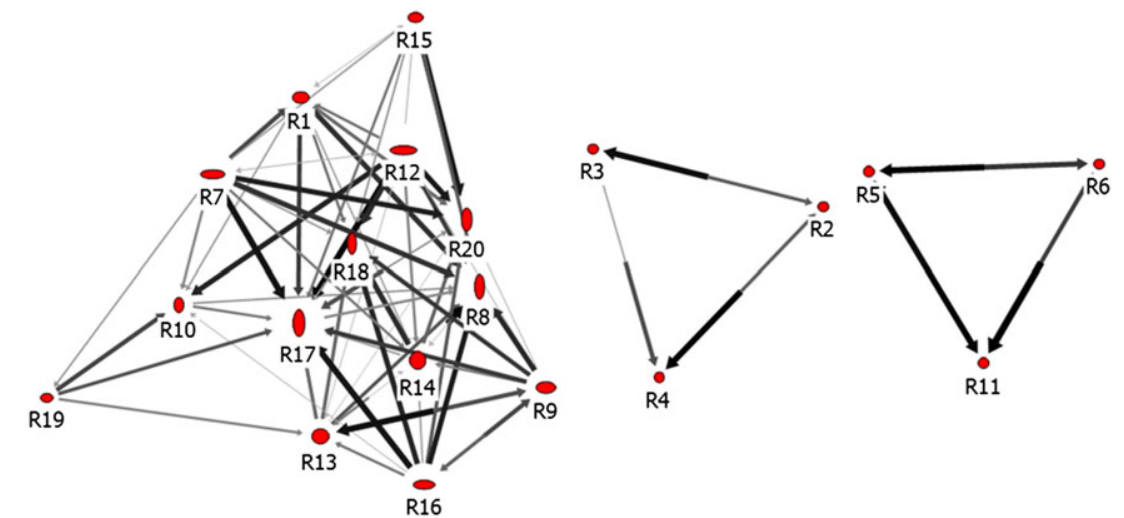

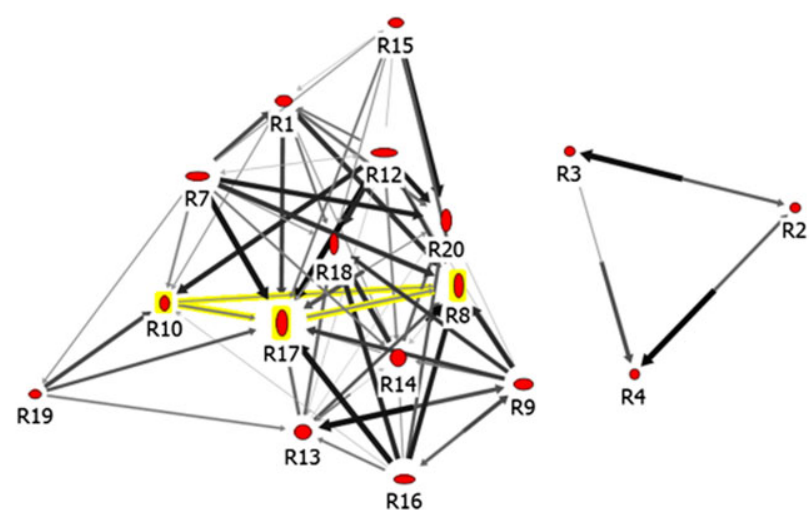

(a) Potential substitutes for R17
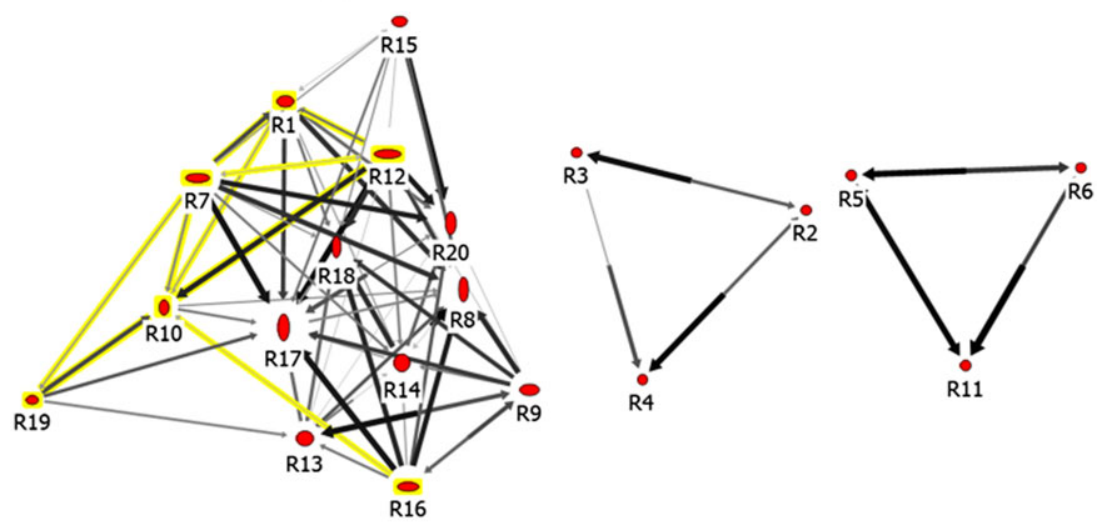

(b) Human resources who can be substituted by R17
FIGURE 5 Highlighted substitution network [Colour figure can be viewed at wileyonlinelibrary.com] shows substitutability between human resources. The network has been simplified to show only the important relationships between human resources. The network shows three groups of substitutable human resources and enables identification of suitable substitutes for each human resource. A highlighted version (Figure 5) of the substitution network identifies R8 and R17 as suitable substitutes for R10 and identifies R10 as a potential substitute for R1, R7, R12, R16, and R19.

Because of asymmetry of substitutability between human resources, the numbers of arcs that a node emits differs from the number of arcs that it receives. These differences are quantified using the in-degree and out-degree of a human resource (Table 7). According to the table, R17, which has the highest in-degree, can replace many other human resources. Thus, a manager responsible for allocating human resources can regard R17 as a multiplayer and incorporate this assessment in the allocation policy. R8, R18, or R20 also have a high in-degree and can be regarded as multiplayers. R18 has the smallest out-degree, so few resources can substitute for him or her. A manager should treat R18 as a critical human resource because critical problems could occur if he/she becomes unavailable (Cabanillas et al., 2015).

The asymmetry of substitutability or the differences between indegree and out-degree of human resources has some important implications. For example, R8 has a high in-degree and low outdegree, so he/she can replace many other workers but not vice versa. The shape of nodes in the substitution network also represents the differences (Figures 4 and 5). A vertical ellipse indicates
TABLE 7 In-degree and out-degree of human resources

\begin{tabular}{|lccc|} 
& In-degree & Out-degree & Differences \\
\hline R1 & 0.064 & 0.130 & -0.065 \\
\hline R2 & 0.043 & 0.048 & -0.005 \\
\hline R3 & 0.044 & 0.044 & 0.000 \\
R4 & 0.047 & 0.042 & 0.004 \\
\hline R5 & 0.049 & 0.047 & 0.002 \\
\hline R6 & 0.044 & 0.050 & -0.006 \\
\hline R7 & 0.021 & 0.217 & -0.196 \\
\hline R8 & 0.245 & 0.043 & 0.203 \\
\hline R9 & 0.065 & 0.154 & -0.089 \\
\hline R10 & 0.108 & 0.043 & 0.065 \\
\hline R11 & 0.050 & 0.046 & 0.004 \\
\hline R12 & 0.021 & 0.240 & -0.219 \\
\hline R13 & 0.108 & 0.128 & -0.020 \\
\hline R14 & 0.147 & 0.111 & 0.036 \\
\hline R15 & 0.042 & 0.108 & -0.066 \\
\hline R16 & 0.022 & 0.176 & -0.154 \\
\hline R17 & 0.265 & 0.063 & 0.202 \\
\hline R18 & 0.196 & 0.021 & 0.175 \\
\hline R19 & 0.021 & 0.065 & -0.044 \\
\hline R20 & 0.219 & 0.045 & 0.174 \\
\hline & & & \\
\hline
\end{tabular}

a human resource who can replace many others but not vice versa; a horizontal ellipse indicates a human resource that can be replaced by many others but not vice versa. 


\subsection{Simulation and results}

A simulation was conducted to demonstrate that the approach is more effective and accurate than existing allocation mechanisms (Table 8) such as role-based allocation.

The simulation model was created based on the event logs presented in the case study. The model generates exceptions randomly then uses three mechanisms to select and allocate substitutes. The model was run 1,000 times to calculate the average total completion time. Descriptive statistics (Table 9) show that the approach outperforms role-based mechanism and random-based mechanisms; these differences were significant (approximately by 5 and $15 \mathrm{hr}$, respectively; Welch's $t$ test; Table 10). Welch's $t$ test was used instead of one-way analysis of variance because the three mechanisms had significantly different variances. Multiple comparisons using GamesHowell post hoc test were conducted to investigate the details of differences between mechanisms (Table 11). The test confirms that the approach outperforms other two methods and enabled rapid and effective responses to various business process exceptions.

TABLE 8 Descriptions of mechanisms

\begin{tabular}{ll} 
Mechanism & \multicolumn{1}{c}{ Description } \\
\hline DoS & $\begin{array}{l}\text { Select substitute based on the proposed approach } \\
\text { Role-based }\end{array}$ \\
$\begin{array}{l}\text { Select substitute randomly among human } \\
\text { resources who share the same role as the } \\
\text { initial performer }\end{array}$ \\
Random-based & Select substitute randomly \\
\hline
\end{tabular}

TABLE 9 Descriptive statistics

\begin{tabular}{lllllll} 
& & & & \multicolumn{2}{l}{$95 \%$ Confidence interval } \\
\cline { 6 - 7 } Mechanism & $N$ & Mean & $\begin{array}{l}\text { Std. } \\
\text { deviation }\end{array}$ & $\begin{array}{l}\text { Lower } \\
\text { bound }\end{array}$ & $\begin{array}{l}\text { Upper } \\
\text { bound }\end{array}$ \\
\hline DoS & 1,000 & 109.54 & 44.87 & 106.76 & 112.33 \\
\hline Role-based & 1,000 & 114.81 & 45.89 & 111.96 & 117.65 \\
\hline Random-based & 1,000 & 124.72 & 57.69 & 121.14 & 128.30 \\
\hline
\end{tabular}

TABLE 10 Welch's $t$ test

\begin{tabular}{llll} 
DF & DF Den & $\boldsymbol{F}$ value & $\boldsymbol{p}$ value \\
2 & $1,976.80$ & 21.57 & 0.000 \\
\hline
\end{tabular}

TABLE 11 Multiple comparisons using Games-Howell test

\begin{tabular}{|c|c|c|c|c|c|c|c|}
\hline \multirow[b]{2}{*}{ Mechanism (i) } & \multirow[b]{2}{*}{ Mechanism (j) } & \multirow{2}{*}{$\begin{array}{c}\text { Mean } \\
\text { difference } \\
(i-j)\end{array}$} & \multirow{2}{*}{$\begin{array}{l}\text { Std. } \\
\text { error }\end{array}$} & \multirow{2}{*}{$\begin{array}{c}t \\
\text { value }\end{array}$} & \multirow{2}{*}{$\begin{array}{c}p \\
\text { value }\end{array}$} & \multicolumn{2}{|c|}{ 95\% Confidence interval } \\
\hline & & & & & & Lower bound & Upper bound \\
\hline Dos & $\begin{array}{l}\text { Role-based } \\
\text { Random-based }\end{array}$ & $\begin{array}{l}-5.26 \\
-15.17\end{array}$ & $\begin{array}{l}2.03 \\
2.31\end{array}$ & $\begin{array}{l}2.59 \\
6.57\end{array}$ & $\begin{array}{l}0.026 \\
0.000\end{array}$ & $\begin{array}{l}-10.01 \\
-20.58\end{array}$ & $\begin{array}{l}-0.51 \\
-9.76\end{array}$ \\
\hline Role-based & $\begin{array}{l}\text { Dos } \\
\text { Random-based }\end{array}$ & $\begin{array}{l}5.26 \\
-9.91\end{array}$ & $\begin{array}{l}2.03 \\
2.33\end{array}$ & $\begin{array}{l}2.59 \\
4.25\end{array}$ & $\begin{array}{l}0.026 \\
0.000\end{array}$ & $\begin{array}{l}0.51 \\
-15.37\end{array}$ & $\begin{array}{l}10.01 \\
-4.45\end{array}$ \\
\hline Random-based & $\begin{array}{l}\text { Dos } \\
\text { Role-based }\end{array}$ & $\begin{array}{l}15.17 \\
9.91\end{array}$ & $\begin{array}{l}2.31 \\
2.33\end{array}$ & $\begin{array}{l}6.57 \\
4.25\end{array}$ & $\begin{array}{l}0.000 \\
0.000\end{array}$ & $\begin{array}{l}9.76 \\
4.45\end{array}$ & $\begin{array}{l}20.58 \\
15.37\end{array}$ \\
\hline
\end{tabular}

Today's organizations must allocate human resources dynamically in response to various business process exceptions such as unavailability of the initial performer or sudden increase of workload. Suitable substitutes who can minimize the effect of the exception must be identified in a systematic and objective way.

Several researchers have suggested the importance of integrating (Cakar, 2003; Glykas, 2011). The rationale behind their arguments is that business processes explicitly specifies the tasks that should be conducted by human resources and provides related data. Processrelated data, such as event logs, often provide information that is useful for decision making in human resource management.

Practitioners are faced with numerous unexpected situations that necessitate rapid reallocation of resources in rapidly changing environments. Often, the biggest challenge is the limited number of available resources, so decision makers require a systematic approach to identify the most suitable alternative performers. The approach will enable delicate and dynamic human resource allocation in such situations. The approach is expected to be especially useful in small and medium enterprises that have few human resources. In addition, the approach will provide objective insights to guide executives and managers when they make decisions related to management of human resources. Often, due to a significant gap between what should happen and what actually happens, many executives or managers have limited information about current circumstances (Song \& van der Aalst, 2008). The approach extracts information from event logs, which record human resources' actual behaviors, so it will increase the accuracy and detail of the information about what is happening. Moreover, all of the information is derived automatically, so it can respond quickly to exceptional situations. Finally, the approach will enable proactive and holistic management of human resources. For example, executives or managers can inspect the network to judge which part of their organization is vulnerable to various types of exceptions, to prepare for these situations in advance, and to redesign organizational structures into more resilient one. SNA techniques that measure structural properties of a given network (e.g., structural holes) can be applied during the judgment. In conclusion, the approach will provide a systematic support to executives and managers in their decisions about management of human resources.

\section{I CONCLUSION}


To address this need, this paper introduced an approach that identifies suitable substitutes by analyzing event logs. The approach applied process mining techniques to event logs to derive profiles of human resources; these profiles show the workers' work experiences in terms of task execution and transfer of work. Then, SNA techniques were used to assess the DoS between human resources. DoS is a novel modification of the Jaccard coefficient. The substitution network summarizes the current state of substitutability between human resources; the manager responsible for allocating human resources can use DoS and substitution network to make a final decision.

The proposed approach can be easily incorporated in current workflow management systems or business process systems to increase the effectiveness and flexibility of human-resource allocation, especially in exceptional situations. If an initial performer of a given task becomes unavailable, the approach can automatically analyze event logs to identify and recommend suitable substitutes for that performer. If the workload of a certain human resource increases suddenly, the approach can automatically create a new instance of his/her work and assign it to a suitable substitute.

Currently, substitutability between human resources is assessed based on frequency. Other dimensions such as time, quality, or cost should also be considered. As future work, an extended approach that considers the time dimension is being developed. Further, additional organizational information, such as department, team, or position, will be considered to increase the accuracy with which substitutes are identified. Finally, the approach will be applied to various organizations to further validate its effectiveness.

\section{ACKNOWLEDGEMENTS}

This work was partially supported by the Business for University Entrepreneurship Center, funded by the Korea Small and Medium Business Administration in 2015 (Grant 10031496). The work was also partially supported by the National Research Foundation of Korea(NRF) grant funded by the Korea Government (MSIT) (NRF-2017R1A2B4008029).

\section{ORCID}

Jooseok Lee (D) https://orcid.org/0000-0001-7791-4503

Seunghoon Lee (i) https://orcid.org/0000-0001-7479-5668

\section{REFERENCES}

Arias, M., Rojas, E., Munoz-Gama, J., \& Sepúlveda, M. (2015). A framework for recommending resource allocation based on process mining. Decision Mining \& Modeling for Business Processes (DeMiMoP), Innsbruck

Cabanillas, C., García, J. M., Resinas, M., Ruiz, D., Mendling, J., \& RuizCortés, A. (2013). Priority-based human resource allocation in business processes. Service-Oriented Computing, Lecture Notes in Computer Science, 8274, 374-388. https://doi.org/10.1007/978-3-642-45005-1. 26

Cabanillas, C., Resinas, M., del-Río-Ortega, A., \& Ruiz-Cortés, A. (2015). Specification and automated design-time analysis of the business process human resource perspective. Information Systems, 52, 55-82. https://doi.org/10.1016/j.is.2015.03.002

Cakar, F. (2003). A business process approach to human resource management. Business Process Management Journal, 9(2), 190-207. https://doi. org/10.1108/14637150310468
Dumas, M., La Rosa, M., Mendling, J., \& Reijers, H. (2013). Fundamentals of business process management. Berlin, Heidelberg: Springer. https://doi. org/10.1007/978-3-642-33143-5

Dustdar, S., \& Hoffmann, T. (2007). Interaction pattern detection in process oriented information systems. Data \& Knowledge Engineering, 62, 138-155. https://doi.org/10.1016/j.datak.2006.07.010

Glykas, M. (2011). Performance measurement in business process, workflow and human resource management. Knowledge and Process Management, 18(4), 241-265. https://doi.org/10.1002/kpm.387

Hanneman, R. A., \& Riddle, M. (2005). Introduction to social network methods. Riverside, CA: University of California. published in digital form at http://faculty.ucr.edu/ hanneman/

Hearn, S., \& Choi, I. (2013). Creating a process and organization fit index: An approach toward optimal process and organization design. Knowledge and Process Management, 20(1), 21-29. https://doi.org/10.1002/ kpm.1400

Huang, Z., Lu, X., \& Duan, H. (2011). Mining association rules to support resource allocation in business process management. Expert Systems with Applications, 38, 9483-9490. https://doi.org/10.1016/j. eswa.2011.01.146

Huang, Z., van der Aalst, W. M. P., Lu, X., \& Duan, H. (2010). An adaptive work distribution mechanism based on reinforcement learning. Expert Systems with Applications, 37, 7533-7541. https://doi.org/10.1016/j. eswa.2010.04.091

Kim, J., Lee, J., Lee, J., \& Choi, I. (2017). An integrated process-related risk management approach to proactive threat and opportunity handling: A framework and rule language. Knowledge and Process Management, 24, 23-37. https://doi.org/10.1002/kpm.1527

Kim, K., Choi, I., \& Park, C. (2011). A rule-based approach to proactive exception handling in business processes. Expert Systems with Applications, 38, 394-409. https://doi.org/10.1016/j.eswa.2010.06.078

Kumar, A., Dijkman, R., \& Song, M. (2013). Optimal resource assignment in workflow for maximizing cooperation. Business Process Management, Lecture Notes in Computer Science, 8094, 235-250. https://doi.org/ 10.1007/978-3-642-40176-3_20

Kumar, A., van der Aalst, W. M. P., \& Verbeek, E. M. W. (2002). Dynamic work distribution in workflow management systems: How to balance quality and performance. Journal of Management Information Systems, 18, 157-193. https://doi.org/10.1080/07421222.2002.11045693

Lee, J., Lee, S., Kim, J., \& Choi, I. (2016). Recommending suitable alternative performers using process mining techniques: Towards smart organizations. The $18^{\text {th }}$ International Conference on Industrial Engineering.

Lee, J., Sung, S., Song, M., \& Choi, I. (2015). A business process simulation framework incorporating the effects of organizational structure. International Journal of Industrial Engineering: Theory Applications and Practice, 22(4), 454-466.

Liu, T., Cheng, Y., \& Ni, Z. (2012). Mining event logs to support workflow resource allocation. Knowledge-Based Systems, 35, 320-331. https:// doi.org/10.1016/j.knosys.2012.05.010

Liu, Y., Wang, J., Yang, Y., \& Sun, J. (2008). A semi-automatic approach for workflow staff assignment. Computers in Industry, 59, 463-476. https://doi.org/10.1016/j.compind.2007.12.002

Ly, L. T., Rinderle, S., Dadam, P., \& Reichert, M. (2006). Mining staff assignment rules from event-based data. Business Process Management Workshops, Lecture Notes in Computer Sciences, 3812, 177-190. https://doi.org/10.1007/11678564_16

Reichert, M., \& Weber, B. (2012). Enabling flexibility in process-aware information systems. Berlin, Heidelberg: Springer. https://doi.org/10.1007/ 978-3-642-30409-5

Russell, N., van der Aalst, W. M. P., Hofstede, A. H. M., \& Edmond, D. (2005). Workflow resource patterns: Identification, representation and tool support. Advanced Information Systems Engineering, Lecture Notes in Computer Science, 3520, 216-232. 
Song, M., \& van der Aalst, W. M. P. (2008). Towards comprehensive support for organizational mining. Decision Support Systems, 46, 300-317. https://doi.org/10.1016/j.dss.2008.07.002

Van der Aalst, W. M. P. (2011). Process mining: Discovery, conformance and enhancement of business processes. Berlin, Heidelberg: Springer. https:// doi.org/10.1007/978-3-642-19345-3

Van der Aalst, W. M. P., Reijers, H., \& Song, M. (2005). Discovering social networks from event logs. Computer Supported Cooperative Work, 14, 549-593. https://doi.org/10.1007/s10606-005-9005-9

Wasserman, S., \& Faust, K. (1994). Social network analysis: Methods and applications. NY: Cambridge University Press. https://doi.org/ 10.1017/CBO9780511815478
Wibisono, A., Nisafani, A. S., Bae, H., \& Park, Y. (2016). A dynamic and human-centric resource allocation for managing business process execution. International Journal of Industrial Engineering: Theory Applications and Practice, 23(4), 270-282.

How to cite this article: Lee J, Lee S, Kim J, Choi I. Dynamic human resource selection for business process exceptions. Knowl Process Manag. 2019;26:23-31. https://doi.org/ 10.1002/kpm.1591 